\title{
Optimal Blind Nonlinear Least-Squares Carrier Phase and Frequency Offset Estimation for Burst QAM Modulations
}

\author{
Yan Wang ${ }^{1}$, Erchin Serpedin ${ }^{1}$ and Philippe Ciblat ${ }^{2}$ \\ ${ }^{1}$ Dept. of Electrical Engineering, Texas A\&M University, College Station, TX 77843, USA \\ ${ }^{2}$ Ecole Nationale Supérieure des Télécommunications, Paris, France
}

\begin{abstract}
This paper introduces a novel family of blind feedforward nonlinear least-squares (NLS) estimators for joint estimation of the carrier phase and frequency offset of quadrature amplitude modulations (QAM). An optimal or "matched" nonlinear estimator that exhibits the smallest asymptotic variance within the family of envisaged NLS-estimators is developed. A class of computationally efficient monomial estimators is also proposed. The asymptotic performance of these estimators is established in closed-form expression and compared with the Cramèr-Rao lower bound corresponding to an unmodulated carrier. Finally, computer simulations are presented to corroborate the theoretical performance analysis.
\end{abstract}

\section{INTRODUCTION}

Quadrature amplitude modulation (QAM) is a highly bandwidth efficient transmission technique for digital communications [10]. One of the problems associated with the burst QAM transmissions is that of carrier acquisition which for efficiency reasons must be performed without the use of a preamble [4].

Recently, a number of blind phase estimators for (square and cross) QAM modulations were reported in [3], [4], [7] and [8], and analyzed in [10] and [11]. They exploit the angle information contained in the fourth or higher-order statistics of the received signal. In this paper, a family of non-data aided (NDA) or blind feedforward nonlinear least-squares (NLS) estimators for joint estimation of carrier phase and frequency offset of carriers that are fully QAM-modulated is proposed and its asymptotic (large sample) performance analyzed in a rigorous way. The proposed NLS estimators exploit a generalized form of the Maximum Likelihood feedforward algorithm, that was originally proposed by A. J. Viterbi and A. M. Viterbi as a blind carrier phase estimator for fully modulated M-PSK transmissions [12], [9]. This carrier phase estimator is referred in the literature as the Viterbi and Viterbi (V\&V) algorithm [7, p. 280]. This paper proposes an optimal or "matched" blind nonlinear carrier estimator for burst QAM modulations that achieves the smallest asymptotic variance within the family of blind NLS estimators. Computationally efficient monomial approximations of the matched estimator are developed, too. The performance of these algorithms is compared with the Cramèr-Rao bound (CRB) of an unmodulated carrier, and shown to exhibit significant improvements over the standard fourth-power estimators proposed in the literature. Furthermore, the proposed family of estimators presents high convergence rates and admits low complexity digital implementations.

\section{PROBLEM FORMULATION}

Consider the baseband representation of an M-QAM modulated signal transmitted through an AWGN channel. Assume that filtering is evenly split between transmitter and receiver so that the overall channel is Nyquist. Filtering the received waveform through a matched filter and sampling at the right time instants yields:

$$
\begin{aligned}
& x(n)=w(n) e^{j \phi(n)}+v(n), \quad n=0, \ldots, N-1, \\
& \phi(n)=\theta+2 \pi F_{e} T n,
\end{aligned}
$$

where $\{w(n)\}$ is the sequence of zero-mean unit variance $\left(\sigma_{w}^{2}:=\mathrm{E}\left\{|w(n)|^{2}\right\}=1\right)$ independently and identically distributed (i.i.d.) M-QAM symbols, $\theta$ and $f_{e}:=F_{e} T$ stand for carrier phase and frequency offset, respectively, $T$ denotes the symbol period, and $\{v(n)\}$ is a zero-mean white Gaussian noise process independent of $w(n)$ and with variance $\sigma_{v}^{2}:=\mathrm{E}\left\{|v(n)|^{2}\right\}$. The Signal-to-Noise Ratio is defined as $\mathrm{SNR}:=10 \log _{10}\left(\sigma_{w}^{2} / \sigma_{v}^{2}\right)$. Due to space limitations, we will present our study only for 4-QAM and 16-QAM constellations. The extension of this work to general square and cross QAM constellations will be reported in a future paper.

As depicted by (1), the problem that we pose is to estimate the unknown phase parameters $\left(\theta\right.$ and $\left.F_{e}\right)$ of a harmonic embedded in unknown multiplicative $(w(n))$ and additive noise $(v(n))$, assuming knowledge of the received samples $\{x(n)\}_{n=0}^{N-1}$. Because the input QAM constellation has quadrant $(\pi / 2)$ symmetry, it follows that the estimates of $\theta$ and $F_{e}$ present 4 -fold ambiguity. Without any loss of generality, we assume that the unknown phase $\theta$ lies in the interval $(-\pi / 4, \pi / 4)$ and $\left|2 \pi f_{e}\right|<\pi / 4$.

The solution that we pursue consists of evaluating first certain generalized moments of the received signal that will remove the unwanted multiplicative effects introduced by the MQAM modulated sequence $w(n)$. It turns out that the resulting problem reduces to the standard problem of estimating the phase parameters of a constant amplitude harmonic embedded in additive noise, for which standard NLS-type estimators can be developed and their asymptotic variance can be established in closed form expression. The key element in deriving the optimal estimator consists of selecting the optimal nonlinear transformation so that the estimator's asymptotic variance is minimized. In the next section, we detail this derivation.

\section{ESTIMATORS FOR 4-QAM CONSTELLATIONS}

\section{A. Matched Nonlinear Carrier Synchronizer}

For 4-QAM constellation, $w(n)$ takes a value from the set $\{\exp (j(\pi / 4+m \pi / 2)), m=0,1,2,3\}$. Consider the polar representation:

$$
x(n)=\rho(n) e^{j \varphi(n)},
$$

and define the process $y(n)$ via the nonlinear transformation:

$$
y(n):=F(\rho(n)) e^{j 4 \varphi(n)},
$$

where $F(\cdot)$ is a general (arbitrary) nonlinear function. 
Conditioned on the transmitted signal $w(n), x(n)$ is normally distributed with the probability density function (pdf) $f(x(n) \mid w(n)=\exp (j(\pi / 4+m \pi / 2)), 0 \leq m \leq 3) \sim$ $\mathcal{N}\left(w(n) \exp (j \phi(n)), \sigma_{v}^{2}\right)$. Throughout the paper, the notation $f(\cdot)$ will stand for the pdf of certain RVs. Due to (2), it follows that:

$$
\begin{aligned}
f(\rho(n), \varphi(n) \mid w(n) & \left.=e^{j\left(\frac{\pi}{4}+\frac{m \pi}{2}\right)}\right)=\frac{\rho(n)}{\pi \sigma_{v}^{2}} e^{-\left(\rho^{2}(n)+1\right) / \sigma_{v}^{2}} \\
& \cdot e^{2 \rho(n) \cos [\varphi(n)-\pi / 4-m \pi / 2-\phi(n)] / \sigma_{v}^{2}} .
\end{aligned}
$$

Based on (4), the joint and marginal pdf of $\rho(n)$ and $\varphi(n)$ take the expressions:

$$
\begin{gathered}
f(\rho(n), \varphi(n))=\frac{1}{4} \sum_{m=0}^{3} f\left(\rho(n), \varphi(n) \mid w(n)=e^{j\left(\frac{\pi}{4}+\frac{m \pi}{2}\right)}\right) \\
=\frac{1}{4} \sum_{m=0}^{3} \frac{\rho(n)}{\pi \sigma_{v}^{2}} e^{-\frac{\rho^{2}(n)+1}{\sigma_{v}^{2}}} e^{\frac{2 \rho(n)}{\sigma_{v}^{2}} \cos \left[\varphi(n)-\frac{\pi}{4}-\frac{m \pi}{2}-\phi(n)\right]} \\
f(\rho(n))=\int_{-\pi}^{\pi} f(\rho(n), \varphi(n)) \mathrm{d} \varphi(n) \\
=\frac{2 \rho(n)}{\sigma_{v}^{2}} e^{-\left(\rho^{2}(n)+1\right) / \sigma_{v}^{2}} I_{0}\left(\frac{2 \rho(n)}{\sigma_{v}^{2}}\right)
\end{gathered}
$$

where $I_{0}(\cdot)$ stands for the zero-order modified Bessel function of the first kind [1, eq. (9.6.16)]. Using (5), some calculations show that:

$$
\begin{aligned}
& \mathrm{E}\{y(n)\}=\mathrm{E}\left\{F(\rho(n)) e^{j 4 \varphi(n)}\right\}=\mathcal{C} e^{j(\pi+4 \phi(n)),} \\
& \mathcal{C}:=|\mathrm{E}\{y(n)\}|=\mathrm{E}\left\{F(\rho(n)) \frac{I_{4}\left(\frac{2 \rho(n)}{\sigma_{v}^{2}}\right)}{I_{0}\left(\frac{2 \rho(n)}{\sigma_{v}^{2}}\right)}\right\},
\end{aligned}
$$

where $I_{k}(\cdot)$ denotes the kth-order modified Bessel function of the first kind [1, eq. (9.6.19)], the expectation in (8) is with respect to (w.r.t.) the marginal distribution of $\rho(n)(6)$ and the resulting amplitude $\mathcal{C}$ is a real constant. Since $w(n)$ and $v(n)$ are i.i.d. and mutually independent, it follows that $u(n):=$ $y(n)-\mathrm{E}\{y(n)\}$ is i.i.d., too. Consequently,

$$
y(n)=\mathcal{C} e^{j(\pi+4 \phi(n))}+u(n), n=0,1, \ldots, N-1,
$$

and $y(n)$ can be viewed as a constant amplitude harmonic embedded in white noise. Note that, in general, $u(n)$ is not circular.

Let $\boldsymbol{\omega}:=\left[\begin{array}{lll}-\mathcal{C} & \omega_{0} & \omega_{1}\end{array}\right]^{T}=\left[\begin{array}{lll}-\mathcal{C} & 4 \theta & 8 \pi f_{e}\end{array}\right]^{T}$, and introduce the following NLS estimator (c.f. [2]):

$$
\begin{aligned}
\hat{\boldsymbol{\omega}} & =\arg \min _{\overline{\boldsymbol{\omega}}} J(\overline{\boldsymbol{\omega}}), \\
J(\overline{\boldsymbol{\omega}}) & =\frac{1}{N} \sum_{n=0}^{N-1}\left|y(n)-\overline{\mathcal{C}} e^{j \sum_{l=0}^{1} \bar{\omega}_{l} n^{l}}\right|^{2} .
\end{aligned}
$$

After some algebra manipulations, the NLS estimates of $\omega_{l}, l=0,1$, are obtained as [2]:

$$
\hat{\omega}_{1}=\arg \max _{\bar{\omega}_{1}} \frac{1}{N}\left|\sum_{n=0}^{N-1} y(n) e^{-j \bar{\omega}_{1} n}\right|^{2},
$$

$$
\hat{\omega}_{0}=\operatorname{angle}\left\{-\sum_{n=0}^{N-1} y(n) e^{-j \hat{\omega}_{1} n}\right\} .
$$

One can observe that the frequency offset estimator can be implemented efficiently by means of the Fast Fourier Transform (FFT) algorithm applied on the sequence $y(n)$. It is wellknown that estimator (10) is asymptotically unbiased and consistent, and also almost asymptotically efficient at high SNR [2] and [5].

Following a procedure similar to the one presented in [2], one can express the asymptotic variances avar $\left(\hat{\omega}_{l}\right)$ of the NLSestimates $\hat{\omega}_{l}, l=0,1$, as:

$$
\begin{aligned}
& \operatorname{avar}\left(\hat{\omega}_{l}\right)=\frac{\mathcal{B}-\mathcal{D}}{\mathcal{C}^{2}} \cdot \frac{1}{2 N^{2 l+1}} \cdot \frac{1}{2 l+1}\left[\frac{(l+2) !}{(l !)^{2}(1-l) !}\right]^{2}, \\
& \mathcal{B}:=\mathrm{E}\left\{|y(n)|^{2}\right\}=\mathrm{E}\left\{F^{2}(\rho(n))\right\} \\
& \mathcal{D}:=\left|\mathrm{E}\left\{y^{2}(n)\right\}\right|=\mathrm{E}\left\{F^{2}(\rho(n)) \frac{I_{8}\left(\frac{2 \rho(n)}{\sigma_{v}^{2}}\right)}{I_{0}\left(\frac{2 \rho(n)}{\sigma_{v}^{2}}\right)}\right\}
\end{aligned}
$$

Next, we determine an optimal or "matched" nonlinearity $F(\cdot)$ which minimizes the asymptotic variance (11). Since in (11), only $\mathcal{B}, \mathcal{C}, \mathcal{D}$ depend on $F(\cdot)$, finding an optimal $F(\cdot)$ resorts to solving the optimization problem:

$$
F_{\min }(\rho(n))=\arg \min _{F} \frac{\mathcal{B}-\mathcal{D}}{\mathcal{C}^{2}} .
$$

Using (8), (12) and (13), $F_{\min }(\cdot)$ is obtained using CauchySchwarz' inequality and admits the expression:

$$
F_{\min }(\rho(n))=\lambda \frac{I_{4}\left(\frac{2 \rho(n)}{\sigma_{v}^{2}}\right)}{I_{0}\left(\frac{2 \rho(n)}{\sigma_{v}^{2}}\right)-I_{8}\left(\frac{2 \rho(n)}{\sigma_{v}^{2}}\right)},
$$

where $\lambda$ is an arbitrary nonzero constant. The asymptotic variances of $\hat{\omega}_{l}, l=0,1$, corresponding to the matched nonlinearity (14) can be expressed as:

$$
\begin{aligned}
& \operatorname{avar}_{\min }\left(\hat{\omega}_{l}\right)=\frac{1}{2 N^{2 l+1}} \cdot \frac{1}{2 l+1} \cdot\left[\frac{(l+2) !}{(l !)^{2}(1-l) !}\right]^{2} \\
& \cdot \frac{1}{\mathrm{E}\left\{\frac{I_{4}^{2}\left(\frac{2 \rho(n)}{\sigma_{v}^{2}}\right)}{I_{0}^{2}\left(\frac{2 \rho(n)}{\sigma_{v}^{2}}\right)-I_{0}\left(\frac{2 \rho(n)}{\sigma_{v}^{2}}\right) I_{8}\left(\frac{2 \rho(n)}{\sigma_{v}^{2}}\right)}\right\}} .
\end{aligned}
$$

\section{B. Monomial Nonlinear Estimators}

As can be observed from (14), $F_{\min }(\rho(n))$ is a function that depends on the SNR. This is not a restrictive requirement since blind SNR estimators that exhibit good performance can be used. However, if estimating the SNR is not desirable, we show next that there exist optimal monomial approximations $\rho^{k}(n), k=0, \ldots, 4$, of the matched nonlinearity $F_{\min }(\rho(n))$ that have almost the same asymptotic variance as (15) and their performance does not necessitate knowledge of the SNR.

It turns out that at high SNRs (SNR $\rightarrow \infty \mathrm{dB})$, based on [9, eq. (15)], the optimal monomial is $G_{h}(\rho(n))=\rho(n)$. Similarly, at low SNRs (SNR $\ll 0 \mathrm{~dB})$, based on [9, eq. (16)], the optimal monomial is $G_{l}(\rho(n))=\rho^{4}(n)$. 
Define the class of processes $y_{k}(n), n=0, \ldots, N$, via the monomial transformations:

$$
y_{k}(n)=\rho^{k}(n) e^{j 4 \varphi(n)}, k=0, \ldots, 4 .
$$

Now it is interesting to study the asymptotic performance of the following class of NLS estimators:

$$
\hat{\boldsymbol{\omega}}^{(k)}=\arg \min _{\overline{\boldsymbol{\omega}}^{(k)}} \frac{1}{N} \sum_{n=0}^{N-1}\left|y_{k}(n)-\overline{\mathcal{C}} e^{j \sum_{l=0}^{1} \bar{\omega}_{l}^{(k)} n^{l}}\right|^{2},
$$

which can be viewed as a special case of (10) and whose asymptotic variances are given by:

$$
\begin{aligned}
& \operatorname{avar}\left(\hat{\omega}_{l}^{(k)}\right)=\frac{\mathcal{B}_{k}-\mathcal{D}_{k}}{\mathcal{C}_{k}^{2}} \cdot \frac{1}{2 N^{2 l+1}} \cdot \frac{1}{2 l+1} \cdot\left[\frac{(l+2) !}{(l !)^{2}(1-l) !}\right]^{2} \\
& \mathcal{B}_{k}:=\mathrm{E}\left\{\left|y_{k}(n)\right|^{2}\right\}=\mathrm{E}\left\{\rho^{2 k}(n)\right\} \\
& \mathcal{C}_{k}:=\left|\mathrm{E}\left\{y_{k}(n)\right\}\right|=\left|\mathrm{E}\left\{\rho^{k}(n) e^{j 4 \varphi(n)}\right\}\right| \\
& \mathcal{D}_{k}:=\left|\mathrm{E}\left\{y_{k}^{2}(n)\right\}\right|=\left|\mathrm{E}\left\{\rho^{2 k}(n) e^{j 8 \varphi(n)}\right\}\right| .
\end{aligned}
$$

Exploiting (6) and [6, eq. (6.643.4)], the following relation was derived in $[12,(\mathrm{~A} 17)]$ :

$$
\mathcal{B}_{k}=\sum_{q=0}^{k}\left(\begin{array}{l}
k \\
q
\end{array}\right)^{2} \sigma_{v}^{2 q} \cdot q !
$$

Using (6), it turns out that:

$\mathrm{E}\left\{y_{k}(n)\right\}=\frac{1}{\alpha^{k}} e^{j(\pi+4 \phi(n))} e^{-\frac{\gamma}{2}} \int_{0}^{\infty} \zeta^{k+1} e^{-\frac{\zeta^{2}}{2}} I_{4}(\alpha \zeta) \mathrm{d} \zeta$,

where: $\alpha:=\sqrt{2} / \sigma_{v}, \gamma:=\alpha^{2}$ and $\zeta:=\alpha \rho(n)$. Based on [6, eq. $(6.643,2)]$ and [1, eq. $(13.1 .32)], \mathcal{C}_{k}$ can be expressed in terms of the confluent hypergeometric function $\Phi(\cdot, \cdot, \cdot)$ :

$$
\mathcal{C}_{k}=\frac{\Gamma\left(\frac{k}{2}+3\right) e^{-\frac{\gamma}{2}}}{\Gamma(5) \sigma_{v}^{4-k}} \Phi\left(\frac{k}{2}+3,5, \frac{\gamma}{2}\right) .
$$

Similarly,

$$
\mathcal{D}_{k}=\frac{\Gamma(k+5) e^{-\frac{\gamma}{2}}}{\Gamma(9) \sigma_{v}^{8-2 k}} \Phi\left(k+5,9, \frac{\gamma}{2}\right) .
$$

Following a similar approach to that presented in [12], one can obtain a slightly more compact expression for the confluent hypergeometric function in (21):

$$
\begin{aligned}
\mathcal{D}_{k}= & \frac{1}{\gamma^{k}}\left[\gamma^{k} \sum_{p=0}^{4+k} p !\left(\begin{array}{c}
4+k \\
p
\end{array}\right)\left(\begin{array}{c}
3-k+p \\
p
\end{array}\right)\left(\frac{-2}{\gamma}\right)^{p}\right. \\
& +(-1)^{5+k} 2^{k} e^{-\frac{\gamma}{2}}\left(\frac{2}{\gamma}\right)^{k+1} \sum_{p=0}^{3-k}\left(\begin{array}{c}
4+k+p \\
p
\end{array}\right) \\
& \left.\cdot \frac{(4+k) !}{(3-k-p) !}\left(\frac{2}{\gamma}\right)^{p}\right], \text { if } k=0,1,2,3, \\
\mathcal{D}_{k}= & 1, \text { if } k=4 .
\end{aligned}
$$

Plugging (19), (20) and (21) back into (18), a closed-form expression for the asymptotic variances $\operatorname{avar}\left(\hat{\omega}_{l}^{(k)}\right)$ is obtained for $k=0, \ldots, 4$ and $l=0,1$. Note that at high SNR $(\rightarrow \infty$ $\mathrm{dB})$, using [1, eq. (13.1.4)], some calculations show that:

$$
\lim _{\mathrm{SNR} \rightarrow \infty} \mathcal{C}_{k}=1
$$

for any $k=0,1, \ldots, 4$. Hence, based on (18), (19), (22) and (23) we obtain:

$$
\lim _{\mathrm{SNR} \rightarrow \infty} \operatorname{avar}\left(\hat{\omega}_{l}^{(k)}\right) \propto \frac{1}{\mathrm{SNR}},
$$

which does not depend on the estimator order $k$, i.e., it turns out that at high SNRs, the performance of estimators (17) for different orders $k$ is asymptotically the same.

\section{ESTIMATORS FOR 16-QAM CONSTELLATIONS}

Following a similar approach to the one presented above, one can develop matched nonlinear estimators for joint estimation of the carrier phase and frequency offset for 16-QAM modulations.

\section{A. Matched Nonlinear Carrier Synchronizer}

Fig. 1 illustrates a representation of a 16-QAM constellation in terms of four 4-QAM constellations, each of them defined by a specific amplitude and phase shift. Thus, for a generic point $w(n)$ of the 16-QAM constellation, it holds that:

$$
\begin{aligned}
& w(n) \in\left\{\frac{1}{\sqrt{5}} e^{j(\pi / 4+m \pi / 2)}, e^{j\left(\eta_{0}+m \pi / 2\right)}, e^{j\left(-\eta_{0}+m \pi / 2\right)},\right. \\
& \left.\frac{3}{\sqrt{5}} e^{j(\pi / 4+m \pi / 2)}, m=0, \cdots, 3\right\}, \eta_{0}:=\tan ^{-1}(1 / 3) .
\end{aligned}
$$

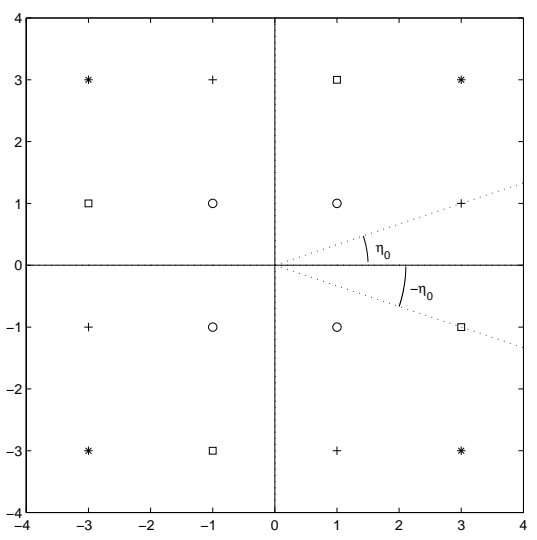

Fig. 1. 16-QAM Constellation

Therefore, we can express the joint and marginal pdf of $\rho(n)$ and $\varphi(n)$ as:

$$
\begin{aligned}
& f(\rho(n), \varphi(n))= \\
& \frac{1}{16} \frac{\rho(n)}{\pi \sigma_{v}^{2}}\left\{\sum_{m=0}^{3} e^{-\frac{1}{\sigma_{v}^{2}}\left(\rho^{2}(n)+\frac{1}{5}\right)} e^{\frac{2 \rho(n)}{\sqrt{5} \sigma_{v}^{2}} \cos \left[\varphi(n)-\frac{\pi}{4}-\frac{m \pi}{2}-\phi(n)\right]}\right. \\
& +\sum_{m=0}^{3} e^{-\frac{1}{\sigma_{v}^{2}}\left(\rho^{2}(n)+\frac{9}{5}\right)} e^{\frac{6 \rho(n)}{\sqrt{5} \sigma_{v}^{2}} \cos \left[\varphi(n)-\frac{\pi}{4}-\frac{m \pi}{2}-\phi(n)\right]}
\end{aligned}
$$




$$
\begin{aligned}
& +\sum_{m=0}^{3} e^{-\frac{1}{\sigma_{v}^{2}}\left(\rho^{2}(n)+1\right)} e^{\frac{2 \rho(n)}{\sigma_{v}^{2}} \cos \left[\varphi(n)-\eta_{0}-\frac{m \pi}{2}-\phi(n)\right]} \\
& \left.+\sum_{m=0}^{3} e^{-\frac{1}{\sigma_{v}^{2}}\left(\rho^{2}(n)+1\right)} e^{\frac{2 \rho(n)}{\sigma_{v}^{2}} \cos \left[\varphi(n)+\eta_{0}-\frac{m \pi}{2}-\phi(n)\right]}\right\} \\
& \\
& f(\rho(n))=\frac{\rho(n)}{2 \sigma_{v}^{2}}\left\{e^{-\frac{1}{\sigma_{v}^{2}}\left(\rho^{2}(n)+\frac{1}{5}\right)} I_{0}\left(\frac{2 \rho(n)}{\sqrt{5} \sigma_{v}^{2}}\right)+\right. \\
& \left.\quad e^{-\frac{1}{\sigma_{v}^{2}}\left(\rho^{2}(n)+\frac{9}{5}\right)} I_{0}\left(\frac{6 \rho(n)}{\sqrt{5} \sigma_{v}^{2}}\right)+2 e^{-\frac{1}{\sigma_{v}^{2}}\left(\rho^{2}(n)+1\right)} I_{0}\left(\frac{2 \rho(n)}{\sigma_{v}^{2}}\right)\right\} .
\end{aligned}
$$

Similarly to the derivations presented in Section III-a, by considering the process $y(n)$ (see eq. (3)), it follows that $y(n)$ can be interpreted as the sum (9). Based on the above expressions, it is not difficult to find that the NLS estimator (10) is asymptotically unbiased and consistent in the presence of 16-QAM constellations, and the asymptotic variances of $\hat{\omega}_{l}, l=0,1$, are still given by $(11)$, where $\mathcal{B}, \mathcal{C}, \mathcal{D}$ take the following expressions:

$$
\begin{aligned}
\mathcal{B} & =\int_{0}^{\infty} F^{2}(\rho(n)) \xi_{1}(\rho(n)) \mathrm{d} \rho(n), \\
\mathcal{C} & =\int_{0}^{\infty} F(\rho(n)) \xi_{2}(\rho(n)) \mathrm{d} \rho(n), \\
\mathcal{D} & =\int_{0}^{\infty} F^{2}(\rho(n)) \xi_{3}(\rho(n)) \mathrm{d} \rho(n),
\end{aligned}
$$

with

$$
\begin{gathered}
\xi_{1}(\rho(n)):=f(\rho(n)), \\
\xi_{2}(\rho(n)):=\frac{\rho(n)}{2 \sigma_{v}^{2}} e^{-\frac{\rho^{2}(n)}{\sigma_{v}^{2}}}\left\{e^{-\frac{1}{5 \sigma_{v}^{2}}} I_{4}\left(\frac{2 \rho(n)}{\sqrt{5} \sigma_{v}^{2}}\right)+\right. \\
\left.e^{-\frac{9}{5 \sigma_{v}^{2}}} I_{4}\left(\frac{6 \rho(n)}{\sqrt{5} \sigma_{v}^{2}}\right)-2 \cos \left(4 \eta_{0}\right) e^{-\frac{1}{\sigma_{v}^{2}}} I_{0}\left(\frac{2 \rho(n)}{\sigma_{v}^{2}}\right)\right\}, \\
\xi_{3}(\rho(n)):=\frac{\rho(n)}{2 \sigma_{v}^{2}} e^{-\frac{\rho^{2}(n)}{\sigma_{v}^{2}}}\left\{e^{-\frac{1}{5 \sigma_{v}^{2}}} I_{8}\left(\frac{2 \rho(n)}{\sqrt{5} \sigma_{v}^{2}}\right)+\right. \\
\left.e^{-\frac{9}{5 \sigma_{v}^{2}}} I_{8}\left(\frac{6 \rho(n)}{\sqrt{5} \sigma_{v}^{2}}\right)+2 \cos \left(8 \eta_{0}\right) e^{-\frac{1}{\sigma_{v}^{2}}} I_{8}\left(\frac{2 \rho(n)}{\sigma_{v}^{2}}\right)\right\} .
\end{gathered}
$$

Using (24)-(26) and the Cauchy-Schwarz' inequality to minimize (11), one can obtain the optimal nonlinearity and its corresponding asymptotic variance:

$$
\begin{aligned}
F_{\text {min }}(\rho(n)) & =\lambda \frac{\xi_{2}(\rho(n))}{\xi_{1}(\rho(n))-\xi_{3}(\rho(n))}, \\
\operatorname{avar}_{\min }\left(\hat{\omega}_{l}\right) & =\frac{1}{2 N^{2 l+1}} \cdot \frac{1}{2 l+1} \cdot\left[\frac{(l+2) !}{(l !)^{2}(1-l) !}\right]^{2} \\
\cdot & \frac{1}{\int_{0}^{\infty} \frac{\xi_{2}^{2}(\rho(n))}{\xi_{1}(\rho(n))-\xi_{3}(\rho(n))} \mathrm{d} \rho(n)} .
\end{aligned}
$$

\section{B. Monomial Nonlinear Estimators}

Unfortunately, due to the complicated form of $\xi_{i}(\rho(n)), i=$ $1,2,3$, it appears difficult to express an optimal approximation of (27) in terms of simple monomial transformations. However, because of their computational efficiency and simplicity, it is still of interest to study the performance of monomial nonlinear estimators for higher-order QAM constellations.

Adopting a similar procedure to the one presented in the previous section, one can find that the asymptotic variances of monomial nonlinear estimates: $\hat{\omega}_{l}^{k}, l=0,1$, and $k=$ $0, \cdots, 4$, in the case of 16-QAM constellations can still be expressed by (18), where:

$$
\begin{aligned}
\mathcal{B}_{k} & =\frac{\sigma_{v}^{2 k}}{4} \sum_{q=0}^{k}\left(\begin{array}{c}
k \\
q
\end{array}\right)^{2} q ! \frac{2 \gamma_{1}^{k-q}+\gamma_{2}^{k-q}+\gamma_{3}^{k-q}}{2^{k-q}}, \\
\mathcal{C}_{k} & =\frac{\sigma_{v}^{k} \Gamma\left(\frac{k}{2}+3\right)}{4 \Gamma(5)}\left[e^{-\frac{\gamma_{2}}{2}}\left(\frac{\gamma_{2}}{2}\right)^{2} \Phi\left(\frac{k}{2}+3,5, \frac{\gamma_{2}}{2}\right)\right. \\
& +e^{-\frac{\gamma_{3}}{2}}\left(\frac{\gamma_{3}}{2}\right)^{2} \Phi\left(\frac{k}{2}+3,5, \frac{\gamma_{3}}{2}\right) \\
& \left.-2 \cos \left(4 \eta_{0}\right) e^{-\frac{\gamma_{1}}{2}}\left(\frac{\gamma_{1}}{2}\right)^{2} \Phi\left(\frac{k}{2}+3,5, \frac{\gamma_{1}}{2}\right)\right], \\
\mathcal{D}_{k} & =\frac{\sigma_{v}^{2 k} \Gamma(k+5)}{4 \Gamma(9)}\left[e^{-\frac{\gamma_{2}}{2}}\left(\frac{\gamma_{2}}{2}\right)^{4} \Phi\left(k+5,9, \frac{\gamma_{2}}{2}\right)\right. \\
& +e^{-\frac{\gamma_{3}}{2}}\left(\frac{\gamma_{3}}{2}\right)^{4} \Phi\left(k+5,9, \frac{\gamma_{3}}{2}\right) \\
& \left.+2 \cos \left(8 \eta_{0}\right) e^{-\frac{\gamma_{1}}{2}}\left(\frac{\gamma_{1}}{2}\right)^{4} \Phi\left(k+5,9, \frac{\gamma_{1}}{2}\right)\right],
\end{aligned}
$$

with $\gamma_{1}:=2 / \sigma_{v}^{2}, \gamma_{2}:=2 / 5 \sigma_{v}^{2}$ and $\gamma_{3}:=18 / 5 \sigma_{v}^{2}$. In the next section, we will compare the performance of monomial nonlinear estimators to that of the optimal estimator (28).

\section{SIMULATION RESULTS}

In this section, we study thoroughly the performance of estimators (10) and (17) for 4-QAM and 16-QAM constellations using computer simulations. The experimental mean-square error (MSE) results of (17) will be compared with the theoretical asymptotic bounds. The experimental results are obtained by performing a number of 200 Monte Carlo trials and the additive noise is generated as zero-mean Gaussian white noise with variance $\sigma_{v}^{2}$.

In this section, we also compare the asymptotic performance of the proposed estimators w.r.t. the CRB of an unmodulated carrier, which has the expression (c.f. [5]):

$$
\operatorname{CRB}\left(\hat{\omega}_{l}\right)=\frac{\sigma_{v}^{2}}{2 N^{2 l+1}} \cdot \frac{1}{2 l+1} \cdot\left[\frac{(l+2) !}{(l !)^{2}(1-l) !}\right]^{2} .
$$

Experiment 1-Asymptotic variances of estimators (14)-(10) and (16)-(17): Figs. 2 and 3 illustrate the theoretical asymptotic variances of estimators (10) and (17) versus SNR. Since the difference between the asymptotic variances of $\hat{\theta}$ and $\hat{F}_{e}$ is just a constant for a given SNR, only the variance of $\hat{F}_{e}$ is plotted. The theoretical asymptotic variances are compared with the CRB. Fig. 2 depicts the performance loss of the asymptotic variances (15) and (18) w.r.t. CRB (i.e., $\left.-10 \log _{10}\left[\operatorname{avar}\left(\hat{\omega}_{l}^{(k)}\right) / \mathrm{CRB}\left(\hat{\omega}_{l}\right)\right]\right)$ for a 4-QAM modulation. It can be seen that the proposed estimators exhibit good accuracy. In high SNR range, their performance coincides with the CRB. 
In low SNR range (near $0 \mathrm{~dB}$ ), the estimators (17) exhibit good performance only for low order nonlinearity orders $(k=1$ and 2 ). From Fig. 2, we can also observe that at high SNRs, the monomial estimators (16)-(17) for different orders $k$ exhibit the same asymptotic variance in the case of 4-QAM constellations. Fig. 3 depicts the asymptotic variances (15) and (18) versus SNR, assuming a 16-QAM constellation and the number of samples $N=500$. Opposed to the results obtained in the case of 4-QAM modulations, the monomial approximation corresponding to $k=4$ appears to exhibit good performance at low SNRs. However, at high SNRs, the optimum nonlinear estimator provides a significant improvement.

Experiment 2-Comparison of MSE of estimators (17) with the theoretical bounds: In Figs. 4 -6, the theoretical bounds (18) are compared with experimental MSEs of the estimators (17). The results are plotted versus SNR in Fig. 4, assuming $N=50$, $\theta=0, F_{e} T=0.011$ and 4-QAM modulation, while in Figs. 56 the following parameters are adopted $N=500, \theta=0.2$, $F_{e} T=0.05$ and 16-QAM constellation. These figures show that for medium and high SNR, the experimental results are well predicted by the asymptotic bounds derived in this paper.

\section{CONCLUSIONS}

In this paper, we have introduced and analyzed a family of blind feedforward joint carrier phase and frequency offset estimators for QAM modulations. A matched nonlinear estimator together with a class of monomial nonlinear estimators were introduced and their performance established. In a future paper, we will extend this work to large dimensional QAM constellations.

Acknowledgement: This work was supported by the NSF Career Award No. CCR-0092901.

\section{REFERENCES}

[1] M. Abramowitz and I. A. Stegun, Eds., Handbook of Mathematical Functions, Washington, DC: National Bureau of Standards, 1964.

[2] O. Besson, M. Ghogho and A. Swami, "Parameter estimation for random amplitude chirp signals," IEEE Trans. Signal Processing, vol. 47, no. 12 pp. 3208-3219, Dec. 1999.

[3] K. V. Cartwright, "Blind phase recovery in general QAM communication systems using alternative higher order statistics," IEEE Signal Processing Letters, vol. 6, no. 12, pp. 327-329, Dec. 1999.

[4] C. N. Georghiades, "Blind carrier phase acquisition for QAM constellations," IEEE Trans. Communications, vol. 45, no. 11, pp. 1477-1486, Nov. 1997.

[5] M. Ghogho, A. K. Nandi and A. Swami, "Cramèr-Rao bounds and maximum likelihood estimation for random amplitude phase-modulated signals," IEEE Trans. Signal Processing, vol. 47, no. 11, pp. 2905-2916, Nov. 1999.

[6] I. S. Gradshteyn and I. M. Ryzhik, Table of Integrals, Series, and Products, New York: Academic, 1965.

[7] U. Mengali and A. N. D' Andrea, Synchronization Techniques for Digital Receivers, Plenum Press, New York, 1997.

[8] M. Moeneclaey and G. de Jonghe, "ML-oriented NDA carrier synchronization for general rotationally symmetric signal constellations," IEEE Trans. Communications, vol. 42, no. 8, pp. 2531-2533, Aug. 1994.

[9] B. E. Paden, "A matched nonlinearity for phase estimation of a PSKmodulated carrier," IEEE Trans. Information Theory, vol. 32, no. 3, pp. 419-422, May 1986.

[10] F. Rice, B. Cowley, B. Moran and M. Rice, "Cramèr-Rao lower bound for QAM phase and frequency estimation," IEEE Trans. Communications, vol. 49 , no. 9 , pp. $1582-1591$, Sept. 2001.

[11] E. Serpedin, P. Ciblat, G. B. Giannakis and P. Loubaton, "Performance analysis of blind carrier phase estimators for general QAM constellations," IEEE Trans. Communications, vol. 49, no. 8, pp. 1816-1823, Aug. 2001.

[12] A. J. Viterbi and A. M. Viterbi, "Nonlinear estimation of PSK-modulated carrier phase with application to burst digital transmissions," IEEE Trans. Information Theory, vol. 29, no. 4, pp. 543-551, July 1983.

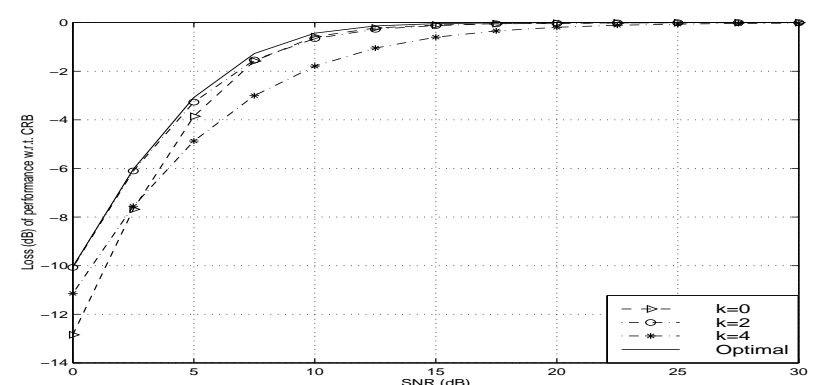

Fig. 2. Performance loss w.r.t. CRB versus SNR for 4-QAM modulation.

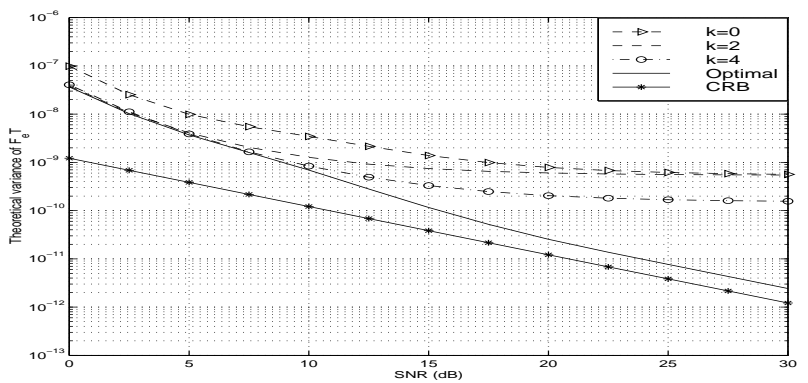

Fig. 3. Theoretical performance of $F_{e} T$ versus SNR for 16-QAM modulation.

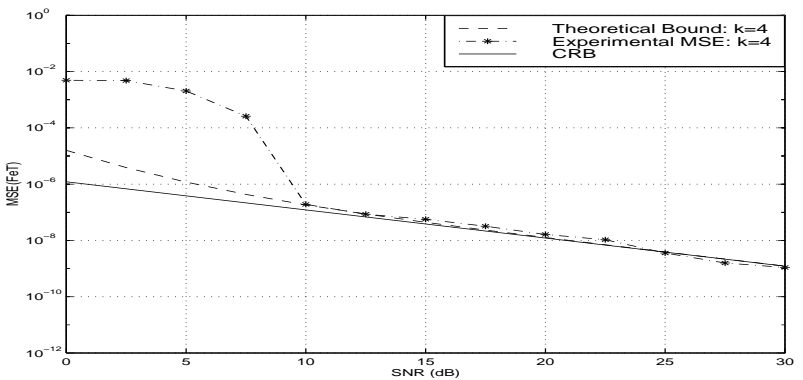

Fig. 4. MSEs of $\widehat{F_{e} T}$ versus SNR for 4-QAM modulation (zero-padding 1024).

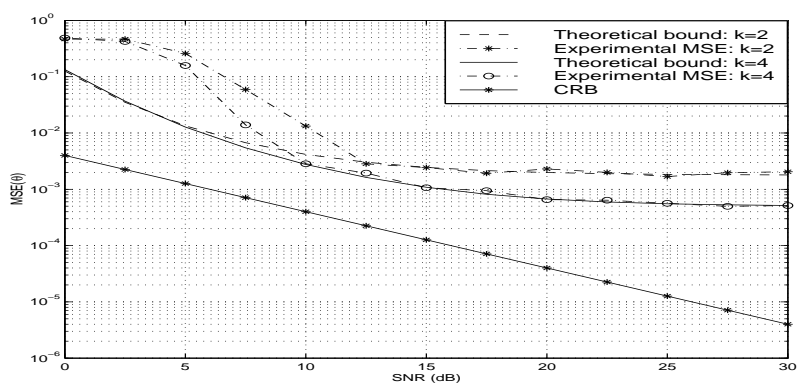

Fig. 5. MSEs of $\widehat{\theta}$ versus SNR for 16-QAM modulation (zero-padding 8192).

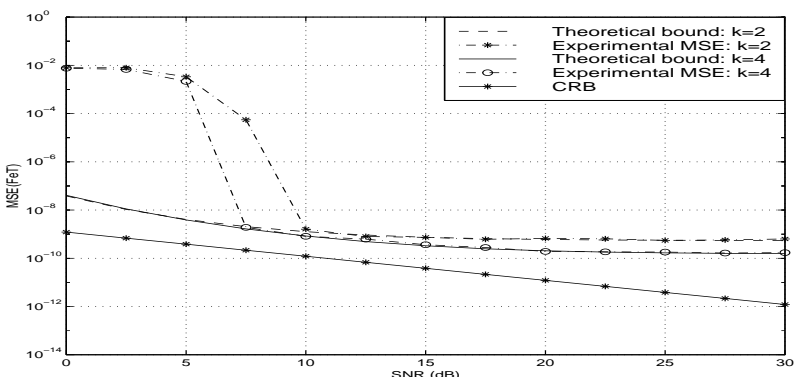

Fig. 6. MSEs of $\widehat{F_{e} T}$ versus SNR for 16-QAM modulation (zero-padding 8192). 